\title{
Three-dimensional CSEM modelling on unstructured tetrahedral meshes using edge finite elements
}

\author{
Octavio Castillo-Reyes, Josep de la Puente, José María Cela \\ Computer Applications in Science \& Engineering, \\ Barcelona Supercomputing Center, \\ Torre Girona, C/ Jordi Girona. 29, 08034 Barcelona, Spain \\ \{octavio.castillo, josep.delapuente, josem.cela\}@bsc.es \\ http://www.bsc.es
}

\begin{abstract}
The last decade has been a period of rapid growth for electromagnetic methods (EM) in geophysics, mostly because of their industrial adoption. In particular, the marine controlled-source electromagnetic method (CSEM) has become an important technique for reducing ambiguities in data interpretation in hydrocarbon exploration. In order to be able to predict the EM signature of a given geological structure, modelling tools provide us with synthetic results which we can then compare to real data. On the other hand and among the modelling methods for EM based upon 3D unstructured meshes, the Nédélec Edge Finite Element Method (EFEM) offers a good trade-off between accuracy and number of degrees of freedom, i.e. size of the problem. Furthermore, its divergence-free basis is very well suited for solving Maxwell's equation. On top of that, we present the numerical formulation and results of 3D CSEM modelling using the Parallel Edge-based Tool for Geophysical Electromagnetic Modelling (PETGEM) on unstructured tetrahedral meshes. We validated our experiments against quasi-analytical results in canonical models.
\end{abstract}

Keywords: CSEM, geophysics, edge finite element, high performance computing.

\section{Introduction}

The electromagnetic methods (EM) are an established tool in geophysics, finding application in many areas such as hydrocarbon and mineral exploration, reservoir monitoring, CO2 storage characterization, geothermal reservoir imaging, water prospecting, and many others. In particular, the marine Controlled-Source Electromagnetic Method (CSEM) has become an important technique for reducing ambiguities in data interpretation in hydrocarbon exploration. In the traditional configuration, the sub-seafloor structure is explored by emitting low frequency signals from a high-powered electric dipole source towed close to the seafloor. By studying the received signal, subsurface structures can be detected at scales of a few tens of meters to depths of several kilometers [2]. 
In order to be able to predict the electromagnetic signature of a given geological body, modelling tools provide us with synthetic results which we can then compare to real data. These tools require a discretisation method in order to obtain an accurate solution to the physical governing equations. As principal discretisation techniques arises the Finite Difference Method (FDM) and Finite Element Method (FEM). In geophysics electromagnetic modelling, the FDM is still the most widely employed discretisation scheme and one of the most practical and highly efficient parallel codes was developed by [1]. However, the main disadvantage of FDM is his incapacity to work with unstructured grids, which limits its use in scenarios where irregular and complex geology has a significant influence or measurements, e.g., a model with strong seabed bathymetry where an imprecise representation could lead to false interpretations.

On the other hand, the FEM supports completely unstructured tetrahedral meshes as well as local refinement, which enables the representation of complex structures and thus improves the solution accuracy. Nevertheless, standard FEM does not correctly take into account all the physical properties of vector fields. In fact, there are three main problems when nodal-based FEM is employed to represent vector fields, namely, occurrence of spurious solutions, inconvenience of imposing boundary conditions at materials interfaces and the difficulty in treating conducting and dielectric edges and corner [13].

Finally, Edge Finite Element Method (EFEM) is free of all the previously mentioned shortcomings because of its uses so-called vector basis functions that assign degrees of freedom (DOFs) to the edges. As a consequence, EFEM meets inherent requirements in geophysical electromagnetic modelling, namely, offers unstructured meshing support, has the ability to eliminate spurious solutions and is claimed to yield accurate results because it's divergence-free basis is well suited for solving Maxwell's equations.

On top of that, we have developed a 3D CSEM tool based upon EFEM for parallel computational architectures: Parallel Edge-based Tool for Geophysical Electromagnetic Modelling (PETGEM). To overcome problems related to the spatial singularity at the source, we have employed a secondary field formulation of Maxwell's equations in their diffusive form, namely, the electric field is decomposed into primary and secondary field. In order to represent complex geological bodies, we use unstructured tetrahedral meshes as these are the easiest to use for very large domains and because offers a good trade-off between accuracy and number of degrees of freedom, i.e. size of the problem.

PETGEM is a Python code for the scalable solution of EM on tetrahedral meshes. It supports parallelism on shared-memory platforms. As result, PETGEM allow users to specify edge-based variational forms of $H($ curl $)$ for the simulation of electromagnetic fields in real 3D CSEM surveys with high accuracy, reliability and efficiency.

In this paper we present the numerical formulation and results of 3D CSEM modelling using PETGEM. It's is divided as follows: Section 2 describes the numerical formulation of 3D CSEM and its role as exploration tool. Section 3 shortly describes the theory associated to EFEM for CSEM applications. In Sec- 
tion 4 we validated our experiments against quasi-analytical results in canonical models. Last section is dedicated to conclusions and future work.

\section{CSEM problem}

Controlled-source Electromagnetic Method (CSEM) is a type of geophysical strategies to study the subsurface electrical conductivity distribution with an ample range of applications. CSEM techniques can be divided into two groups depending on the domain in which collected data is interpreted: time domains (TDEM) or frequency domains (FDEM). In the case of oil prospecting, marine CSEM surveys are done predominantly using FDEM [15].

In marine 3D CSEM, also referred as seabed logging [11] or CSEM, a deeptowed electric dipole transmitter is used to produce a low frequency electromagnetic signal (primary field) which interacts with the electrically conductive Earth and induces eddy currents that become sources of a new electromagnetic signal (secondary field). The two fields, the primary and the secondary one, add up to a resultant field, which is measured by remote receivers placed on the seabed. Since the electromagnetic field at low frequencies, for which displacement currents are negligible, depends mainly on the electric conductivity distribution of the ground, it is possible to detect thin resistive layers beneath the seabed by studying the received signal [16]. Operating frequencies of transmitters in CSEM may range between 0.1 and $10 \mathrm{~Hz}$, and the choice depends on the dimensions of a model. In most studies, typical frequencies vary from 0.25 to $1 \mathrm{~Hz}$, which means that for source-receiver offsets of $10-12 \mathrm{~km}$, the penetration depth of the method can extend to several kilometres below the seabed [12], [16].

The main disadvantage of CSEM is its relatively low resolution compared to seismic imaging. Therefore, CSEM is often used in conjunction with seismic surveying as the latter helps to constrain the resistivity model. Figure 1 depicts the CSEM.

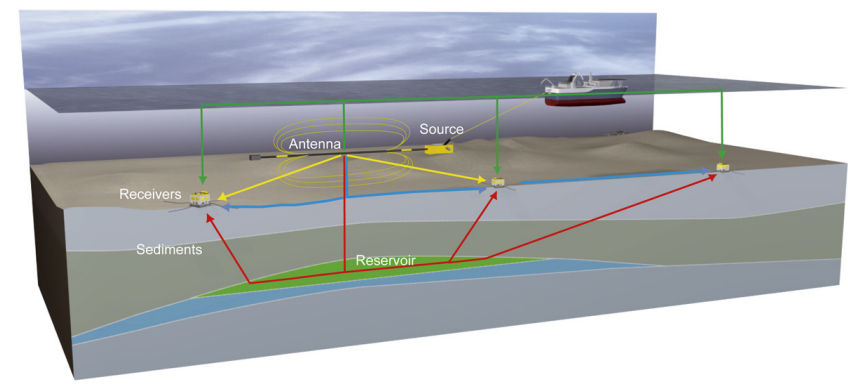

Fig. 1. Controlled-source Electromagnetic Method (CSEM) 
3D CSEM is nowadays a well-known geophysical prospecting tool in the offshore environment and a commonplace in industry, examples of that can be found in [2], [7], [9], [10], [18].

3D CSEM modelling is typically solved in frequency domain, which involves the numerical solution of Maxwell's equations in stationary regimes for heterogeneous anisotropic electrically conductive domains. As already mentioned, CSEM surveys generally work with low frequency electromagnetic fields $(\sim 1 \mathrm{~Hz})$ because the electric conductivity of the geological structures is much larger than their dielectric permittivity. As a consequence, in an unbound domain $\Gamma$, the electric field can be obtained by solving Maxwell's equations in their diffusive form:

$$
\begin{gathered}
\nabla \times \mathbf{E}=i \omega \mu_{0} \mathbf{H} \\
\nabla \times \mathbf{H}=\mathbf{J}_{s}+\tilde{\sigma} \mathbf{E}
\end{gathered}
$$

where we have ommited the harmonic time dependence $e^{-i \omega t}$, with $\omega$ is the angular frequency, $\mu_{0}$ the free space magnetic permeability, $\mathbf{J}_{s}$ the distribution of source current, $\tilde{\sigma} \mathbf{E}$ the induced current in the conductive Earth and $\tilde{\sigma}$ the electrical conductivity which is assumed isotropic for simplicity.

In numerical approximations of EM fields there are two main drawbacks. The first one is the inevitable spatial singularity at the source. The second is the grid refinement requirements in order to capture the rapid change of the primary field [3]. In order to mitigate these issues, we used a secondary field approach where the total electric field $\mathbf{E}$ is obtained as:

$$
\begin{aligned}
& \mathbf{E}=\mathbf{E}_{p}+\mathbf{E}_{s} \\
& \tilde{\sigma}=\tilde{\sigma}_{s}+\Delta \tilde{\sigma}
\end{aligned}
$$

where subscripts $p$ and $s$ represent a primary field and secondary field respectively. For a general layered Earth model, $\mathbf{E}_{p}$ can be computed semi-analytically by using Hankel transform filters. Based on this decomposition and following the work by [17] the equation system to solve $\mathbf{E}_{s}$ is:

$$
\nabla \times \nabla \times \mathbf{E}_{s}+i \omega \mu \tilde{\sigma} \mathbf{E}_{s}=-i \omega \mu \Delta \sigma \mathbf{E}_{p}
$$

where the electrical conductivity $\sigma$ is a function of position that is allowed to vary in $3 \mathrm{D}$, whereas the vacuum permeability $\mu$ is set to the free space value $\mu_{0}$. We set homogeneous Dirichlet boundary conditions, $\mathbf{E}_{s}=0$ on $\partial \Gamma$. The range of applicability of this conditions can be determined based on the skin depth of the electric field [19]. 


\section{Edge finite element method}

For the computation of $\mathbf{E}_{s}$, we have implemented the Nédélec EFEM which uses vector basis functions defined on the edges of the corresponding elements. Its vector basis functions are divergence-free but not curl-free [13]. Thus, EFEM naturally ensures tangential continuity and allows normal discontinuity of $\mathbf{E}_{s}$ at material interfaces. In our approach we used unstructured tetrahedral meshes because of their ability to represent complex geological structures such as bathymetry or reservoirs as well as the local refinement capability in order to improve the solution accuracy. Figure 2 shows the tetrahedral Nédélec elements (lowest order) together with their node and edge indexing.

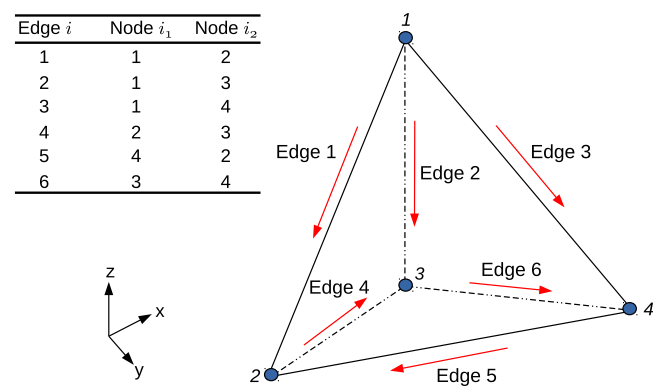

Fig. 2. Tetrahedral Nédélec edge element with node/edge indexing.

We assign the tangential component of the secondary electric field to the edges in the mesh. Therefore, all components of the electric field at a point $\mathbf{x}$ located inside a tetrahedral element $e$ can be obtained as follows:

$$
\mathbf{E}^{e}(\mathbf{x})=\sum_{i=1}^{6} \mathbf{N}_{i}^{e}(\mathbf{x}) E_{i}^{e}
$$

where $\mathbf{N}_{i}^{e}$ are the vector basis functions associated to each edge $i$ and $E_{i}^{e}$ their degrees of freedom. Considering the node and edge indexing in figure 2, the vector basis functions can be expressed as follows:

$$
\mathbf{N}_{i}^{e}=\left(\lambda_{i 1}^{e} \nabla \lambda_{i 2}^{e}-\lambda_{i 2}^{e} \nabla \lambda_{i 1}^{e}\right) \ell_{i}^{e}
$$

where subscripts $i 1$ and $i 2$ are the first and second nodes linked to the $i$-th edge, $\lambda_{i}^{e}$ are the linear nodal basis functions, and $\ell_{i}^{e}$ is the length of the $i$-th edge of the element $e$.

By substituting expression (6) into (5), and using Galerkin's approach, the weak form of the original differential equation becomes:

$$
Q_{i}=\int_{\Omega} \mathbf{N}_{i} \cdot\left[\nabla \times \nabla \times \mathbf{E}_{s}-i \omega \mu \tilde{\sigma} \mathbf{E}_{s}+i \omega \mu \Delta \tilde{\sigma} \mathbf{E}_{p}\right] d V
$$


The compact discretized form of (8) is obtained after applying the Green's theorem:

$$
\left[K_{j k}^{e}+i \omega \tilde{\sigma}_{e} M_{j k}^{e}\right] \cdot\left\{E_{s k}\right\}=-i \omega \mu \Delta \tilde{\sigma}_{e} R_{k}^{e}
$$

where $K^{e}$ and $M^{e}$ are the elemental stiffness and mass matrices which can be calculated analytically or numerically [13] and $R_{k}^{e}$ is the right hand side which requires numerical integration. Our formulation does not requires calculate the jacobian matrix because the construction of elemental matrices is performed in real space. The interested reader will find a rigorous mathematical development of $K^{e}$ and $M^{e}$ in [4], [5], [13],

In our experiments, the numerical solution of the system of linear equations was obtained using a quasi-minimum residual (QMR) method without preconditioner.

\section{Results}

We validated our EFEM formulation and PETGEM solution against the quasi analytical results of the canonical model by [7]. PETGEM code is developed as open-source at Computer Applications in Science \& Engineering (CASE) of the Barcelona Supercomputing Center - Centro Nacional de Supercomputación. The interested reader will find a comprehensive description about PETGEM design and capabilities in [5].

Model described in [7] consists in four-layers: $1000 \mathrm{~m}$ thick seawater $(3.3$ $S / m), 1000 \mathrm{~m}$ thick sediments $(1 \mathrm{~S} / \mathrm{m}), 100 \mathrm{~m}$ thick oil $(0.01 \mathrm{~S} / \mathrm{m})$ and 1400 m thick sediments $(1 S / m)$. Our computational domain is a $[0,3500]^{3} \mathrm{~m}$ cube. Figure 3 shows a $3 \mathrm{D}$ view of the unstructured tetrahedral mesh for the halfspace $y>1750$, with the color scale representing the electrical conductivity $\sigma$ for each layer.

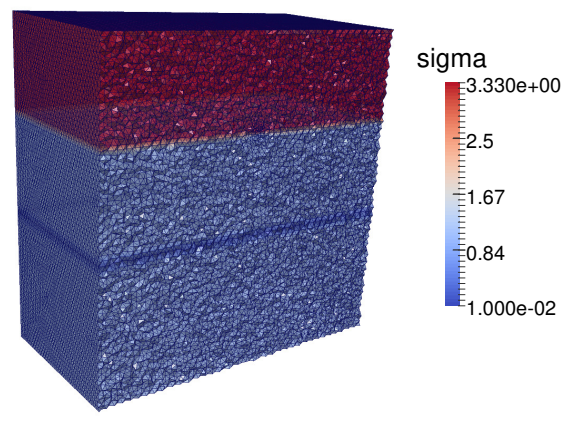

Fig. 3. Unstructured tetrahedral mesh for $y>1750$.

For this model we used a $1 \mathrm{~Hz}$ x-directed dipole source as in [4] which is located at $z=975 \mathrm{~m}, x=1750 \mathrm{~m}$ and $y=1750 \mathrm{~m}$. The receivers are placed in- 
line to the source position and along its orientation, directly above the seafloor $(z=990)$. In order to validate the approach, we have prepared a set of hierarchically refined meshes in order to verify the convergence of the obtained solution. For all cases the mesh has been locally refined around the source region.

Figure 4 shows a comparison of the x-component of total electric field between our EFEM solution and the quasi-analytical solution obtained with the WHAM tool [14]. In figure 4 it is easy to see the effect of our imperfect absorbing boundaries which can be mitigated by enlargening the domain with element sizes increasing logarithmically outwards from the zone of interest. The total electric field in figure 4 was calculated using a mesh with $\approx 12$ millions of edges (degrees of freedom).

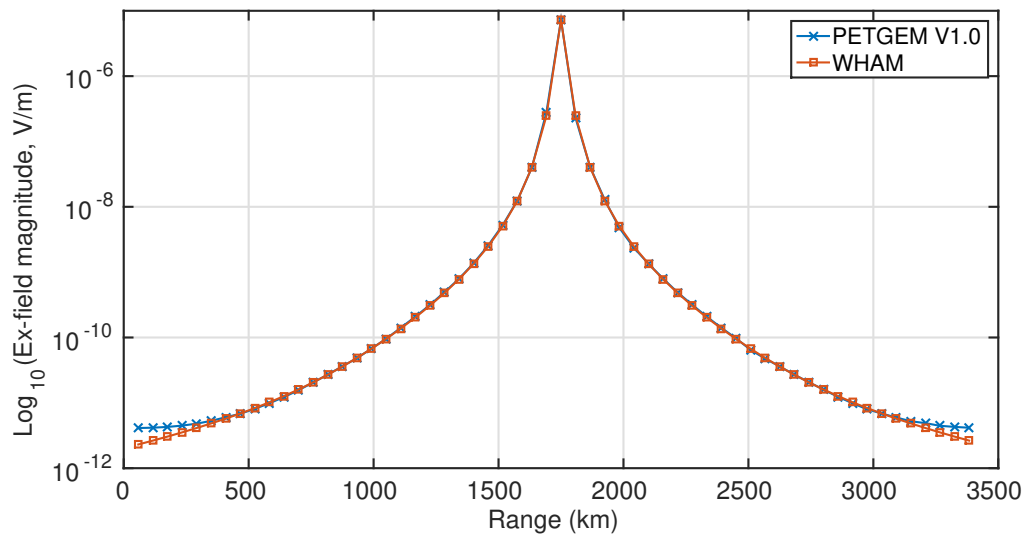

Fig. 4. Total electric field comparative for x-component

Using WHAM as reference solution and excluding those receivers closest to the boundaries, we have quantified the errors in our resulting electric fields by means of the $L^{1}, L^{2}$ and $L^{i n f}$ for our set of meshes, as plotted in figure 5 . Degrees of freedom, mesh spacing and errors for each mesh are depicted in table 1 . We can observe the expected linear convergence in our scheme for all error norms and mesh sizes.

\begin{tabular}{|c|c|c|c|c|}
\hline DOFs & $h(m)$ & $L^{1}$ & $L^{2}$ & $L^{i n f}$ \\
\hline Mesh $16.17 \times 10^{4}$ & $2.0 \times 10^{2}$ & $2.8447 \times 10^{-7}$ & $2.5059 \times 10^{-7}$ & $2.4939 \times 10^{-7}$ \\
\hline Mesh $24.36 \times 10^{5}$ & $1.0 \times 10^{2}$ & $1.6652 \times 10^{-7}$ & $1.0365 \times 10^{-7}$ & $9.0489 \times 10^{-8}$ \\
\hline Mesh $33.43 \times 10^{6}$ & $5.0 \times 10^{1}$ & $1.2859 \times 10^{-7}$ & $8.9955 \times 10^{-8}$ & $7.3234 \times 10^{-8}$ \\
\hline Mesh $41.19 \times 10^{7}$ & $3.3 \times 10^{1}$ & $1.5615 \times 10^{-8}$ & $8.3129 \times 10^{-8}$ & $7.0474 \times 10^{-8}$ \\
\hline
\end{tabular}

Table 1. Summary of results for convergence test 


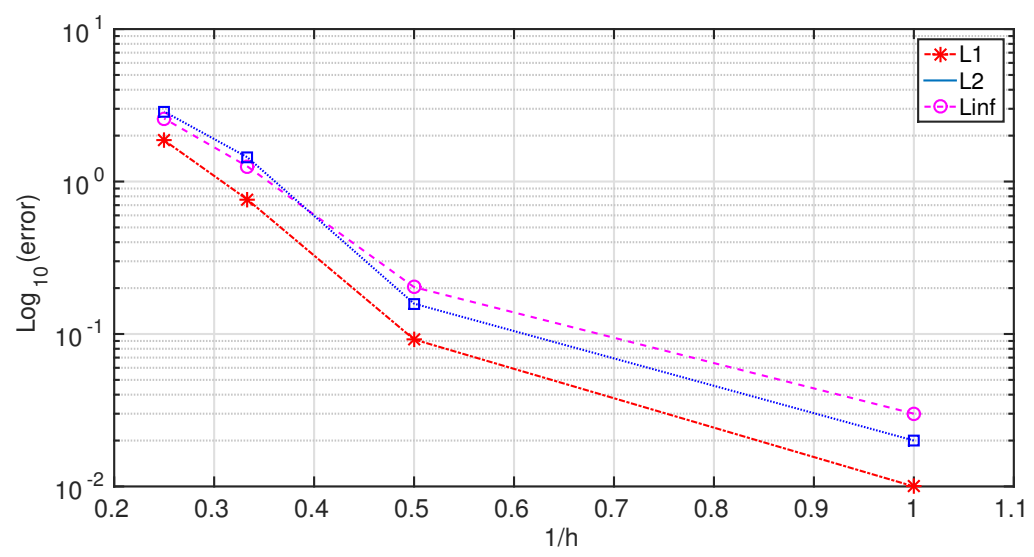

Fig. 5. Convergence order in $L^{1}, L^{2}$ and $L^{i n f}$ norm.

Finally, in table 2 we include some information about the algorithmic effort using a QMR solver for all cases.

\begin{tabular}{|c|c|c|}
\hline & iterations & $\|r\|$ \\
\hline Mesh 1 & 1776 & $9.9777 \times 10^{-7}$ \\
\hline Mesh 2 & 3468 & $9.9703 \times 10^{-7}$ \\
\hline Mesh 3 & 5512 & $9.7994 \times 10^{-7}$ \\
\hline Mesh 4 & 8986 & $1.8174 \times 10^{-6}$ \\
\hline
\end{tabular}

Table 2. Summary of results for a QMR solver

\subsection{Conclusions}

We have studied the feasibility of 3D CSEM modelling on unstructured tetrahedral meshes using Nédélec EFEM. The formulation is interesting because of its low degree of freedom count and natural divergence-free property. We have employed a secondary field formulation to overcome problems related to the spatial singularity at the source. In our examples, a simple QMR solver was sufficed to obtain accurate solutions. Our formulation was validated against a canonical model of an off-shore hydrocarbon reservoir. The PETGEM solution of this model shows a good agreement with the quasi-analytical results in canonical models. The numerical results also demonstrate convergence to the reference solution. Thus, we conclude that our modelling scheme is capable of computing reliable results for 3D CSEM scenarios. Future work will aim at including other features such as seafloor bathymetry and anisotropy to the scheme as well as 
comparing the behaviour of the PETGEM with other modelling approaches for CSEM.

Acknowledgments. Authors of this work has received funding from the European Union's Horizon 2020 research and innovation programme under the Marie-Sklodowska Curie grant agreement No. 644202.

The research leading to these results has received funding from the European Union's Horizon 2020 Programme (2014-2020) and from Brazilian Ministry of Science, Technology and Innovation through Rede Nacional de Pesquisa (RNP) under the HPC4E Project (www.hpc4e.eu), grant agreement No. 689772.

Authors gratefully acknowledge the support from the Mexican National Council for Science and Technology (CONACyT). Numerical tests in this work were performed on the MareNostrum supercomputer of the Barcelona Supercomputing Center - Centro Nacional de Supercomputación (www.bsc.es).

\section{References}

1. Alumbaugh, D., Newman, G., Prevost, L., Shadid, J.: Three-dimensional wideband electromagnetic modeling on massively parallel computers. Wiley Online Library, $1,1-23(1996)$

2. Boulaenko, M., Hesthammer, J., Vereshagin, A., Gelting, P., Davies, R., Wedberg, T.: Marine CSEM TechnologyThe Luva Case. Houston Geological Society (2007)

3. Cai, H., Xiong, B., Han, M., Zhdanov, M.,: 3D controlled-source electromagnetic modeling in anisotropic medium using edge-based finite element method. Computers \& Geosciences, 73, 164-176 (2014)

4. Castillo-Reyes, O., de la Puente, J., Puzyrev, V., Cela, J. M.: Edge-based electric field formulation in 3D CSEM simulations: a parallel approach. Proceedings of the 6th International Conference and Workshop on Computing and Communication, IEEE (2015)

5. Castillo-Reyes, O., de la Puente, J., Modesto, D., Puzyrev, V., Cela, J. M.: Parallel tool for numerical approximation of 3D electromagnetic surveys in geophysics. Computacin y Sistemas, Thematic issue: Topic Trends in Computing Research in Catalonia. 20, 1, 29-39 (2016)

6. Chung, Y., Son, J. S., Lee, T. J., Kim, H. J., Shin, C.: Three-dimensional modelling of controlled-source electromagnetic surveys using an edge finite-element method with a direct solver. Geophysical Prospecting, 32, 6, 1468-1483 (2014)

7. Constable, S., Weiss, C.: Mapping thin resistors and hydrocarbons with marine EM methods: Insights from 1D modeling. Geophysics, 71, 2, G43-G51 (2006)

8. Constable, S.: Marine electromagnetic methodsA new tool for offshore exploration. The Leading Edge. 25, 4, 438-444 (2006)

9. Constable, S., Srnka, L. J.: An introduction to marine controlled-source electromagnetic methods for hydrocarbon exploration. Geophysics, 72, 2, WA3-WA12 (2007)

10. Constable, S.: Ten years of marine CSEM for hydrocarbon exploration. Geophysics, $75,5,75 \mathrm{~A} 67-75 \mathrm{~A} 81(2010)$

11. Eidesmo, T., Ellingsrud, S., MacGregor, L. M., Constable, S., Sinha, M. C., Johansen, S. E., Kong, F. N., Westerdahl, H.: Sea bed logging (SBL), a new method 
for remote and direct identification of hydrocarbon filled layers in deepwater areas. First break: Society of Exploration Geophysicists. 20, 3, 144-152 (2002)

12. Hanif, N., Hussain, N., Yahya, N., Daud, H., Yahya, N., Noh, M.: 1D Modeling of Controlled-Source Electromagnetic (CSEM) Data using Finite Element Method for Hydrocarbon Detection in Shallow Water. Proceedings of the International MultiConference of Engineers and Computer Scientists. (2011)

13. Jianming, J.: The Finite Element Method in Electromagnetics. Wiley (2002)

14. Key, K.: 1D inversion of multicomponent, multifrequency marine CSEM data: Methodology and synthetic studies for resolving thin resistive layers. Geophysics, F9-F20 (2009)

15. Key, K.: Marine electromagnetic studies of seafloor resources and tectonics. Surveys in geophysics. 33, 1, 135-167 (2012)

16. Koldan, J.: Numerical solution of 3-D electromagnetic problems in exploration geophysics and its implementation on massively parallel computers. Polytechnic University of Catalonia (2013)

17. Newman, G., Alumbaugh, D.: Three-dimensional induction logging problems, Part 2: A finite-difference solution. Geophysics, 67, 2, 484-491 (2002)

18. Orange, A., Key, K., Constable, S.: The feasibility of reservoir monitoring using time-lapse marine CSEM. Geophysics, 74, 2, F21-F29 (2009)

19. Puzyrev, V., Koldan, J., de la Puente, J., Houzeaux, G., Vázquez, M., Cela, J. M.: A parallel finite-element method for three-dimensional controlled-source electromagnetic forward modelling. Geophysical Journal International, ggt027 (2013) 